\title{
PERSISTENT URETHRITIS ASSOCIATED WITH STAGHORN CALCULUS OF THE KIDNEY*
}

\author{
BY
}

\author{
R. D. CATTERALL \\ fames Pringle House, The Middlesex Hospital, London, W.1.
}

Urethritis associated with urinary calculi is of rare occurrence. Harkness (1950) described cases of urethritis secondary to stones in the urethra, bladder, prostate and Cowper's glands. $\mathrm{He}$ also gave the history of a patient who developed acute non-specific urethritis, which was cured without active treatment when a renal stone was passed spontaneously after an attack of ureteric colic. Since then occasional cases of urethritis and stones in the renal tract have been reported in the literature and authors have considered it worth while to report single cases because the association has been considered to be unusual.

\section{Case Report}

A 36-year-old computer consultant developed persistent urethritis, oedema of the penis, and a staghorn calculus of the kidney. In 1965, at the age of 33 years, he had a single episode of haematuria. He was investigated at St. Peter's Hospital for Urological Diseases, London, but no cause was found. An intravenous pyelogram and cystoscopy showed nothing abnormal.

In April, 1967, he developed a watery, purulent urethral discharge and had an episode of terminal haematuria; the discharge was diagnosed as nonspecific urethritis and treatment was given with tetracycline. Because this treatment did not produce any improvement, he attended James Pringle House of the Middlesex Hospital in May, 1967.

Examination.-He was found to have a watery, purulent, urethral discharge, oedema of the external urinary meatus, and tenderness with slight enlargement of the lymph nodes in both groins.

Progress.-A diagnosis of non-specific urethritis was made and he was treated with oxytetracycline by mouth. Cultures of the discharge and the urine both grew $B$. proteus. The discharge persisted and in view of the sensitivities of the organisms he was treated with ampicillin and later with nitrofurantoin but without any improvement.

$\star$ Received for publication December 9, 1968.
The discharge persisted throughout the next 6 months despite treatment and $B$. proteus was grown on several occasions from both the discharge and the urine. By November, 1967, the external urinary meatus was very red and oedematous and there was a considerable

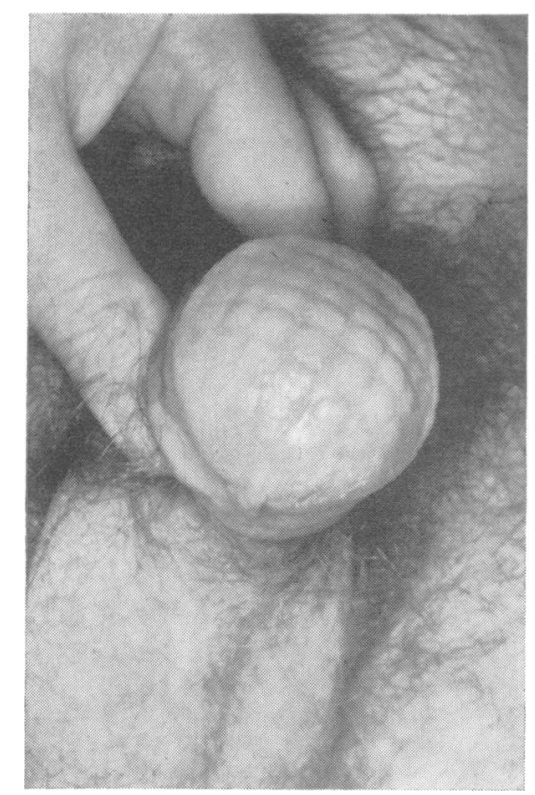

FIo. 1.-Oedema of glans penis with persistent urethritis due to $B$. proteus.

swelling of the glans penis with pitting oedema (Fig. 1). The discharge was copious and watery and he was obliged to wear a cotton wool pad in his underpants. Proteus mirabilis was grown from the urethral discharge and was sensitive to ampicillin and streptomycin. Three early morning specimens of urine were examined for acid-fast bacilli with negative results. A long course of ampicillin was prescribed and the amount of discharge 
decreased but never completely disappeared.

By January, 1968, the oedema of the glans was worse. The discharge persisted and $B$. proteus was again cultured. An intravenous pyelogram revealed a large staghorn calculus in the right renal pelvis (Fig. 2).

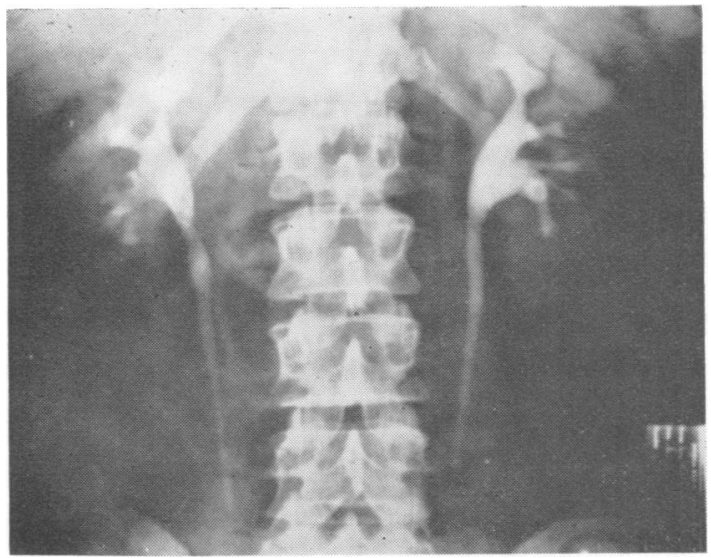

FIG. 2.-Staghorn calculus of kidney.

There was some dilatation of the right ureter and the bladder wall was thickened. The blood urea was $28 \mathrm{mg}$. per cent.; serum calcium and phosphorus were normal.

Operation.-Early in February, 1968, the calculus was removed from the right kidney (by $\mathrm{Mr}$. Richard Turner-Warwick).

Result.-The patient made an uneventful recovery and was kept on a maintenance dose of ampicillin for one month. The urethral discharge disappeared within a few days of the operation and has not recurred since. The redness of the urinary meatus and the oedema of the glans penis slowly improved and by May, 1968, there had been a marked improvement.

When he was last seen in September, 1968, there was no discharge, the urine in the two-glass test was macroscopically clear, but there was still some residual swelling of the glans penis.

\section{Discussion}

It is well known that persistent urinary infection favours the formation of urinary calculi. Organisms such as $B$. proteus and Staphylococcus albus are capable of splitting urea with the formation of an alkaline urine in which phosphatic calculi are prone to develop. Staghorn calculi, which fill the renal pelvis and calyces, are frequently quite symptomless and may be discovered only by chance in radiographs of the abdomen. This patient was known to have had a normal intravenous pyelogram $2 \frac{1}{2}$ years before the discovery of the staghorn calculus. It is difficult to be sure whether his urethritis was secondary to a descending infection of the urinary tract with $B$. proteus or whether the original infection was in the urethra, resulting in an ascending infection leading to stone formation. The oedema of the glans penis was probably due to chronic inflammation of the penile lymphatic vessels, and the residual oedema, after the urethritis had disappeared, suggests that some permanent damage had been done to the lymphatics.

The association of urethritis and urinary calculi has been described in the past; but the development of a staghorn calculus of the kidney in a patient with a persistent urethral discharge due to $B$. proteus, in whom cure of the urethritis followed removal of the calculus, must be rare. I have been unable to find the report of any similar case in the literature.

\section{Summary}

A case of persistent urethritis associated with $B$. proteus infection and staghorn calculus formation is described; the condition failed to respond to antibiotic treatment until the calculus was discovered and removed.

\section{REFERENCE}

HARKNESS, A. H. (1950). "Non-gonococcal Urethritis", pp. 159, 219. Livingstone, Edinburgh.

\section{Urétrite persistante associée à un calcul en corne de cerf du rein}

\section{RÉSUMÉ}

Un cas d'urétrite persistante associée à une infection à $B$. proteus et à la présence d'un calcul rénal en "corne de cerf" est rapporté. Cette urétrite ne répondit au traitement antibiotique qu'après la découverte et l'exérèse du calcul. 